For Internal Distribution Only

Accelerator Division

Alternating Gradient Synchrotron Department

BROOKHAVEN NATIONAL LABORATORY

Upton, New York 11973

Accelerator Division

Technical Note

AGS/AD/Tech. Note No. 439

Projections of Beam Distributions

C.J. Gardner

July 11, 1996 


\title{
Projections of Beam Distributions
}

\author{
C. J. Gardner
}

July 11, 1996

\section{Introduction}

In the following note some basic results of Linear Algebra are applied to obtain the projections of beam distributions. Projections of the ellipsoid which contains the beam are considered first and then Gaussian distributions are treated.

\section{The Beam Ellipsoid}

Let $x_{0}, x_{0}^{\prime}, y_{0}, y_{0}^{\prime}$ be the horizontal and vertical positions and angles (or canonical momenta) of a beam particle with respect to the reference trajectory at a point $s_{0}$ along the trajectory. Then the positions, $x$ and $y$, and angles, $x^{\prime}$ and $y^{\prime}$, of the particle at the point $s$ along the trajectory are given by

$$
\mathbf{Z}=\mathbf{T} \mathbf{Z}_{0}
$$

where

$$
\mathbf{Z}=\left(\begin{array}{c}
Z_{1} \\
Z_{2} \\
Z_{3} \\
Z_{4}
\end{array}\right)=\left(\begin{array}{l}
x \\
x^{\prime} \\
y \\
y^{\prime}
\end{array}\right), \quad \mathbf{Z}_{0}=\left(\begin{array}{c}
x_{0} \\
x_{0}^{\prime} \\
y_{0} \\
y_{0}^{\prime}
\end{array}\right)
$$

and $\mathbf{T}$ is the four-by-four transfer matrix between $s_{0}$ and $s$. The matrix $\mathbf{T}$ is symplectic and therefore has unit determinant.

Now suppose we define an ellipsoid at $s_{0}$ by

$$
\mathbf{Z}_{0}^{\dagger} \mathbf{E}_{0}^{-1} \mathbf{Z}_{0}=\epsilon
$$


where $\mathbf{E}_{0}^{-1}$ is a four-by-four real, symmetric, positive-definite matrix with unit determinant. (We use a $\dagger$ to denote the transpose of a vector or matrix.) Then it is easy to show that the ellipsoid at $s_{0}$ is transformed into another ellipsoid at $s$. Using $\mathbf{Z}_{0}=\mathbf{T}^{-1} \mathbf{Z}$ in (3) we find

$$
\mathbf{Z}^{\dagger} \mathbf{E}^{-1} \mathbf{Z}=\epsilon
$$

where

$$
\mathbf{E}=\mathbf{T} \mathbf{E}_{0} \mathbf{T}^{\dagger}
$$

Equation (4) defines an ellipsoid provided the matrix $\mathbf{E}^{-1}$ is symmetric and positive-definite. Since $\mathbf{E}_{0}$ is symmetric $\left(\mathbf{E}_{0}^{\dagger}=\mathbf{E}_{0}\right)$ we have

$$
\mathbf{E}^{\dagger}=\mathbf{T} \mathbf{E}_{0}^{\dagger} \mathbf{T}^{\dagger}=\mathbf{T} \mathbf{E}_{0} \mathbf{T}^{\dagger}=\mathbf{E}
$$

and $\mathbf{E}^{-1}$ is therefore symmetric. Now a real and symmetric matrix, $\mathbf{A}$, is positive-definite if the quadratic form $\mathbf{Z}^{\dagger} \mathbf{A Z}>\mathbf{0}$ for every $\mathbf{Z} \neq \mathbf{0}$. To show that $\mathbf{E}^{-1}$ is positive-definite, consider

$$
\mathbf{Z}^{\dagger} \mathbf{E}^{-1} \mathbf{Z}=\mathbf{Z}_{0}^{\dagger} \mathbf{T}^{\dagger} \mathbf{E}^{-1} \mathbf{T} \mathbf{Z}_{0}=\mathbf{Z}_{0}^{\dagger} \mathbf{E}_{\mathbf{0}}^{-1} \mathbf{Z}_{0}
$$

Since $\mathbf{Z}_{0}^{\dagger} \mathbf{E}_{0}^{-1} \mathbf{Z}_{0}>0$ for all $\mathbf{Z}_{0} \neq \mathbf{0}$, and since $\mathbf{Z}=\mathbf{0}$ if and only if $\mathbf{Z}_{0}=\mathbf{0}$, it follows that $\mathbf{Z}^{\dagger} \mathbf{E}^{-1} \mathbf{Z}>\mathbf{0}$ for all $\mathbf{Z} \neq \mathbf{0}$. Therefore $\mathbf{E}^{-1}$ is positive-definite, and the ellipsoid defined by $\mathbf{E}_{0}$ is transformed into another ellipsoid defined by $\mathbf{E}$ in going from $s_{0}$ to $s$. We note that since $|\mathbf{T}|=1$,

$$
|\mathbf{E}|=|\mathbf{T}|\left|\mathbf{E}_{0}\right|\left|\mathbf{T}^{\dagger}\right|=\left|\mathbf{E}_{0}\right|
$$

and the volume enclosed by the ellipsoid is therefore conserved.

Now since $\mathbf{Z}^{\dagger} \mathbf{E}^{-1} \mathbf{Z}<\epsilon$ if and only if $\mathbf{Z}_{0}^{\dagger} \mathbf{E}_{0}^{-1} \mathbf{Z}_{0}<\epsilon$, we see that any particle inside (outside) the ellipsoid (3) at $s_{0}$ will be inside (outside) the ellipsoid (4) at $s$. Thus if we define the beam ellipsoid to be the smallest ellipsoid which contains the beam particles at $s_{0}$, then the evolution of this ellipsoid provides a convenient way of keeping track of the motion of the beam without having to track each particle individually.

\section{Maximum Extent in each Dimension}

Let us now determine the maximum extent, in each dimension, of the ellipsoid defined by (4). Since the matrix, $\mathbf{E}$, is real and symmetric there 
exists an orthogonal transformation $\left(\mathbf{O O}^{\dagger}=\mathbf{I}\right)$ which diagonalizes $\mathbf{E}$. Thus

$$
\mathbf{O E O}^{\dagger}=\mathbf{e}=\left(\begin{array}{llll}
e_{1} & 0 & 0 & 0 \\
0 & e_{2} & 0 & 0 \\
0 & 0 & e_{3} & 0 \\
0 & 0 & 0 & e_{4}
\end{array}\right)
$$

and

$$
\mathbf{E}=\mathbf{O}^{\dagger} \mathbf{e O}, \quad \mathbf{E}^{-1}=\mathbf{O}^{\dagger} \mathbf{e}^{-1} \mathbf{O} .
$$

Since $\mathbf{E}$ is positive-definite, its eigenvalues (the matrix elements $e_{i}$ ) are all positive, and it follows that

$$
E_{i i}=\sum_{j, k} O_{j i} e_{j k} O_{k i}=\sum_{k}\left(O_{k i}\right)^{2} e_{k}>0,
$$

i.e. the diagonal elements of $\mathbf{E}$ are all positive. Now let

$$
\mathbf{Y}=\mathbf{O Z}, \quad \mathbf{Z}=\mathbf{O}^{\dagger} \mathbf{Y} .
$$

Then we have

$$
\epsilon=\mathbf{Z}^{\dagger} \mathbf{E}^{-1} \mathbf{Z}=\mathbf{Y}^{\dagger} \mathbf{O} \mathbf{E}^{-1} \mathbf{O}^{\dagger} \mathbf{Y}=\mathbf{Y}^{\dagger} \mathbf{e}^{-1} \mathbf{Y}
$$

and the equation of the ellipsoid in the transformed coordinates, $Y_{i}$, is

$$
\epsilon=\sum_{i} Y_{i}^{2} / e_{i}
$$

It follows that the maximum extent of each coordinate $Y_{i}$ is given by

$$
Y_{i}^{2} \leq \epsilon e_{i}
$$

To obtain the maximum extent of the coordinate $Z_{i}$, we write

$$
Z_{i}=\sum_{j} O_{j i} Y_{j}=\sum_{j}\left(\sqrt{e_{j}} O_{j i}\right)\left(Y_{j} / \sqrt{e_{j}}\right) .
$$

Then making use of the Schwarz inequality [1] we have

$$
Z_{i}^{2} \leq \sum_{j}\left(O_{j i} \sqrt{e_{j}}\right)^{2} \sum_{j}\left(Y_{j} / \sqrt{e_{j}}\right)^{2}
$$

and using (11) and (14) in (17) we have

$$
Z_{i}^{2} \leq \epsilon E_{i i} .
$$


Thus the diagonal elements of $\mathbf{E}$ give the maximum extent in each dimension of the ellipsoid defined by $\mathbf{Z}^{\dagger} \mathbf{E}^{-1} \mathbf{Z}=\epsilon$.

The equality in (17) and (18) holds if and only if $Y_{j} / \sqrt{e_{j}}=\lambda O_{j i} \sqrt{e_{j}}$, which is true if and only if

$$
Y_{j}=\lambda O_{j i} e_{j} .
$$

Using this equation in (14) we find

$$
\epsilon=\lambda^{2} \sum_{j}\left(O_{j i}\right)^{2} e_{j}=\lambda^{2} E_{i i}
$$

and therefore

$$
\lambda^{2}=\epsilon / E_{i i} .
$$

Then using (12), (19) and (10) we find

$$
Z_{k}=\sum_{j} O_{j k} Y_{j}=\lambda \sum_{j} O_{j k} e_{j} O_{j i}=\lambda E_{k i}
$$

and therefore

$$
Z_{i}=\lambda E_{i i}= \pm \sqrt{\epsilon E_{i i}}, \quad Z_{k}=\left(E_{k i} / E_{i i}\right) Z_{i}
$$

Thus when $Z_{i}^{2}=\epsilon E_{i i}$ the other components of $\mathbf{Z}$ are given by the second of equations (23).

\section{Projections of the Ellipsoid}

We can learn more about the size and shape of the ellipsoid by examining its projections onto the $\left(x, x^{\prime}\right),\left(y, y^{\prime}\right)$ and $(x, y)$ planes. To obtain the projections we partition the matricies in equations (1) and (5) into two-by-two matricies. A discussion of the algebra of partitioned matricies may be found in Refs. $[2,3]$. Introducing the notation

$$
\mathbf{E}_{0}=\left(\begin{array}{ll}
\mathbf{F}_{0} & \mathbf{C}_{0} \\
\mathbf{C}_{0}^{\dagger} & \mathbf{G}_{0}
\end{array}\right), \quad \mathbf{E}=\left(\begin{array}{ll}
\mathbf{F} & \mathbf{C} \\
\mathbf{C}^{\dagger} & \mathbf{G}
\end{array}\right), \quad \mathbf{T}=\left(\begin{array}{cc}
\mathbf{M} & \mathbf{n} \\
\mathbf{m} & \mathbf{N}
\end{array}\right)
$$

and

$$
\mathbf{Z}_{0}=\left(\begin{array}{c}
\mathbf{X}_{0} \\
\mathbf{Y}_{0}
\end{array}\right), \quad \mathbf{X}_{0}=\left(\begin{array}{c}
x_{0} \\
x_{0}^{\prime}
\end{array}\right), \quad \mathbf{Y}_{0}=\left(\begin{array}{c}
y_{0} \\
y_{0}^{\prime}
\end{array}\right)
$$




$$
\mathbf{Z}=\left(\begin{array}{c}
\mathbf{X} \\
\mathbf{Y}
\end{array}\right), \quad \mathbf{X}=\left(\begin{array}{l}
x \\
x^{\prime}
\end{array}\right), \quad \mathbf{Y}=\left(\begin{array}{l}
y \\
y^{\prime}
\end{array}\right)
$$

where $\mathbf{F}_{0}, \mathbf{G}_{0}, \mathbf{C}_{0}, \mathbf{F}, \mathbf{G}, \mathbf{C}, \mathbf{M}, \mathbf{N}, \mathbf{m}, \mathbf{n}$ are two-by-two matricies, the equation $\mathbf{E}=\mathbf{T} \mathbf{E}_{0} \mathbf{T}^{\dagger}$ becomes

$$
\begin{aligned}
\mathbf{F} & =\mathbf{M} \mathbf{F}_{0} \mathbf{M}^{\dagger}+\mathbf{n} \mathbf{G}_{0} \mathbf{n}^{\dagger}+\mathbf{n} \mathbf{C}_{0}^{\dagger} \mathbf{M}^{\dagger}+\mathbf{M} \mathbf{C}_{0} \mathbf{n}^{\dagger}, \\
\mathbf{G} & =\mathbf{N} \mathbf{G}_{0} \mathbf{N}^{\dagger}+\mathbf{m} \mathbf{F}_{0} \mathbf{m}^{\dagger}+\mathbf{N} \mathbf{C}_{0}^{\dagger} \mathbf{m}^{\dagger}+\mathbf{m} \mathbf{C}_{0} \mathbf{N}^{\dagger}, \\
\mathbf{C} & =\mathbf{M} \mathbf{C}_{0} \mathbf{N}^{\dagger}+\mathbf{n} \mathbf{C}_{0}^{\dagger} \mathbf{m}^{\dagger}+\mathbf{M} \mathbf{F}_{0} \mathbf{m}^{\dagger}+\mathbf{n} \mathbf{G}_{0} \mathbf{N}^{\dagger}
\end{aligned}
$$

and $\mathbf{Z}=\mathbf{T} \mathbf{Z}_{0}$ becomes

$$
\mathbf{X}=\mathbf{M} \mathbf{X}_{\mathbf{0}}+\mathbf{n} \mathbf{Y}_{0}, \quad \mathbf{Y}=\mathbf{m X}_{0}+\mathbf{N Y}_{\mathbf{0}} .
$$

Now consider the ellipsoid $\mathbf{Z}_{0}^{\dagger} \mathbf{E}_{0}^{-1} \mathbf{Z}_{0}=\epsilon$. To find the projection onto the $x_{0}, x_{0}^{\prime}$ plane we seek a transformation, $\mathbf{T}$, from coordinates given by $\mathbf{X}_{0}$, $\mathbf{Y}_{0}$ to new coordinates given by $\mathbf{X}, \mathbf{Y}$ such that $\mathbf{X}=\mathbf{X}_{0}$ and the equation for the transformed ellipsoid is of the form

$$
\mathbf{X}^{\dagger} \mathbf{F}^{-1} \mathbf{X}+\mathbf{Y}^{\dagger} \mathbf{G}^{-1} \mathbf{Y}=\epsilon
$$

where $\mathbf{F}^{-1}$ and $\mathbf{G}^{-1}$ are positive-definite. We obtain such a transformation if we choose

$$
\mathbf{M}=\mathbf{N}=\mathbf{I}, \quad \mathbf{n}=\mathbf{0}, \quad \mathbf{m}=-\mathbf{C}_{0}^{\dagger} \mathbf{F}_{0}^{-1}
$$

Then we have

$$
\mathbf{T}=\left(\begin{array}{cc}
\mathbf{I} & \mathbf{0} \\
\mathbf{m} & \mathbf{I}
\end{array}\right), \quad \mathbf{T}^{-1}=\left(\begin{array}{cc}
\mathbf{I} & \mathbf{0} \\
-\mathbf{m} & \mathbf{I}
\end{array}\right)
$$

and the equation $\mathbf{E}=\mathbf{T} \mathbf{E}_{0} \mathbf{T}^{\dagger}$ becomes

$$
\mathbf{F}=\mathbf{F}_{0}, \quad \mathbf{C}=0, \quad \mathbf{G}=\mathbf{G}_{0}-\mathbf{C}_{0}^{\dagger} \mathbf{F}_{0}^{-1} \mathbf{C}_{0} .
$$

Thus

$$
\mathbf{E}^{-1}=\left(\begin{array}{ll}
\mathbf{F}_{0}^{-1} & \mathbf{0} \\
\mathbf{0} & \mathbf{G}^{-1}
\end{array}\right)
$$

and the equation for the transformed ellipsoid is

$$
\mathbf{Z}^{\dagger} \mathbf{E}^{-1} \mathbf{Z}=\mathbf{X}_{0}^{\dagger} \mathbf{F}_{0}^{-1} \mathbf{X}_{0}+\mathbf{Y}^{\dagger} \mathbf{G}^{-1} \mathbf{Y}=\epsilon
$$


where

$$
\mathbf{Y}=\mathbf{Y}_{0}-\mathbf{C}_{0}^{\dagger} \mathbf{F}_{0}^{-1} \mathbf{X}_{0}
$$

Now since $\mathbf{E}_{0}^{-1}$ is positive-definite and since $\mathbf{T}^{-1}$ exists, the matrix $\mathbf{E}^{-1}$ is positive-definite. The quadratic forms $\mathbf{X}_{0}^{\dagger} \mathbf{F}_{0}^{-1} \mathbf{X}_{0}$ and $\mathbf{Y}^{\dagger} \mathbf{G}^{-1} \mathbf{Y}$ are therefore positive-definite and it follows that

$$
\mathbf{X}_{0}^{\dagger} \mathbf{F}_{0}^{-1} \mathbf{X}_{0} \leq \epsilon
$$

Defining

$$
\mathbf{f}_{0}=\mathbf{F}_{0} / D, \quad D=\left|\mathbf{F}_{0}\right|^{1 / 2}
$$

we obtain

$$
\mathbf{X}_{0}^{\dagger} \mathbf{f}_{0}^{-1} \mathbf{X}_{0} \leq \epsilon D, \quad\left|\mathbf{f}_{0}\right|=1
$$

which defines an elliptical region of area $\pi \epsilon D$. This region is the projection of the ellipsoid $\mathbf{Z}_{0}^{\dagger} \mathbf{E}_{0}^{-1} \mathbf{Z}_{0}=\epsilon$ onto the $x_{0}, x_{0}^{\prime}$ plane. The boundry of this region is given by

$$
\mathbf{X}_{0}^{\dagger} \mathbf{F}_{0}^{-1} \mathbf{X}_{0}=\epsilon
$$

For these points we have

$$
\mathbf{Y}^{\dagger} \mathbf{G}^{-1} \mathbf{Y}=\mathbf{0}, \quad \mathbf{Y}=\mathbf{0}
$$

and therefore

$$
\mathbf{Y}_{0}=\mathbf{C}_{0}^{\dagger} \mathbf{F}_{0}^{-1} \mathbf{X}_{0}
$$

Thus equations (41) and (43) give the points on the surface of the ellipsoid which project onto the boundry of the $x_{0}, x_{0}^{\prime}$ projection.

The argument given above may be extended to higher dimensions by appropriately partitioning the matricies $\mathbf{E}_{0}, \mathbf{E}$, and $\mathbf{T}$. For the case of $\mathbf{a}$ six-dimensional ellipsoid, the projection onto the $x_{0}, x_{0}^{\prime}$ plane can be obtained by partitioning the matricies as follows:

$$
\mathbf{F}=\left(\begin{array}{cc}
E_{11} & E_{12} \\
E_{21} & E_{22}
\end{array}\right), \quad \mathbf{C}=\left(\begin{array}{cccc}
E_{13} & E_{14} & E_{15} & E_{16} \\
E_{23} & E_{24} & E_{25} & E_{26}
\end{array}\right)
$$

and

$$
\mathbf{C}^{\dagger}=\left(\begin{array}{ll}
E_{13} & E_{23} \\
E_{14} & E_{24} \\
E_{15} & E_{25} \\
E_{16} & E_{26}
\end{array}\right), \quad \mathbf{G}=\left(\begin{array}{cccc}
E_{33} & E_{34} & E_{35} & E_{36} \\
E_{43} & E_{44} & E_{45} & E_{46} \\
E_{53} & E_{54} & E_{55} & E_{56} \\
E_{63} & E_{64} & E_{65} & E_{66}
\end{array}\right)
$$


with similar partitions for $\mathbf{E}_{0}$ and $\mathbf{T}$. Similarly, if we let

$$
\mathbf{F}=\left(E_{11}\right), \quad \mathbf{C}=\left(\begin{array}{lllll}
E_{12} & E_{13} & E_{14} & E_{15} & E_{16}
\end{array}\right)
$$

and.

$$
\mathbf{C}^{\dagger}=\left(\begin{array}{l}
E_{12} \\
E_{13} \\
E_{14} \\
E_{15} \\
E_{16}
\end{array}\right), \quad \mathbf{G}=\left(\begin{array}{lllll}
E_{22} & E_{23} & E_{24} & E_{25} & E_{26} \\
E_{32} & E_{33} & E_{34} & E_{35} & E_{36} \\
E_{42} & E_{43} & E_{44} & E_{45} & E_{46} \\
E_{52} & E_{53} & E_{54} & E_{55} & E_{56} \\
E_{62} & E_{63} & E_{64} & E_{65} & E_{66}
\end{array}\right)
$$

with similar partitions for $\mathbf{E}_{0}$ and $\mathbf{T}$, then the projection of the ellipsoid onto the $x_{0}$ axis is given by equations (38-43) with $\mathrm{X}_{0}=x_{0}$ and $\mathbf{F}_{0}=\left(\mathbf{E}_{0}\right)_{11}=D$. This provides an alternate proof of the results of Section 3.

Generalizing the preceeding arguments we find that the projection of the ellipsoid, $\mathbf{Z}^{\dagger} \mathbf{E}^{-1} \mathbf{Z}=\epsilon$, on the $Z_{m}, Z_{n}$ plane is the elliptical region

$$
\mathbf{X}^{\dagger} \mathbf{f}^{-1} \mathbf{X} \leq \epsilon D
$$

where

$$
\mathbf{X}=\left(\begin{array}{c}
Z_{m} \\
Z_{n}
\end{array}\right), \quad D=\left(E_{m m} E_{n n}-E_{m n}^{2}\right)^{1 / 2},
$$

and the matrix elements of $\mathbf{f}$ are

$$
f_{11}=E_{m m} / D, \quad f_{22}=E_{n n} / D, \quad f_{12}=f_{21}=E_{m n} / D
$$

\section{Projections of the Gaussian Distribution}

Now consider the four-dimensional Gaussian distribution,

$$
\rho\left(x_{0}, x_{0}^{\prime}, y_{0}, y_{0}^{\prime}\right)=\rho\left(\mathbf{Z}_{0}\right)=\left(\frac{1}{2 \pi \epsilon}\right)^{2} e^{-W_{0}}
$$

where

$$
W_{0}=\frac{1}{2 \epsilon} \mathbf{Z}_{0}^{\dagger} \mathbf{E}_{0}^{-1} \mathbf{Z}_{0}, \quad\left|\mathbf{E}_{0}\right|=1
$$

Let us first show that the normalization of the distribution is such that

$$
N=\int \rho\left(\mathbf{Z}_{0}\right) d x_{0} d x_{0}^{\prime} d y_{0} d y_{0}^{\prime}=1 .
$$


Transforming to coordinates, $\mathbf{Y}=\mathbf{O Z}_{0}$, which diagonalize $\mathbf{E}_{0}$ as in Section 3 , we have

$$
N=\left(\frac{1}{2 \pi \epsilon}\right)^{2} \int e^{-W_{0}} d Y_{1} d Y_{2} d Y_{3} d Y_{4}
$$

where

$$
W_{0}=\frac{1}{2 \epsilon} \sum_{i} Y_{i}^{2} / e_{i}
$$

and

$$
e_{1} e_{2} e_{3} e_{4}=\left|\mathbf{E}_{0}\right|=1
$$

Then using the integral

$$
\int_{-\infty}^{+\infty} e^{-A^{2} Y^{2}} d Y=\sqrt{\pi} / A
$$

we obtain

$$
N=\left(\frac{1}{2 \pi \epsilon}\right)^{2}(\sqrt{2 \pi \epsilon})^{4} \sqrt{e_{1} e_{2} e_{3} e_{4}}=1 .
$$

Now using the results of Section 4 we can readily obtain the projections of the distribution onto the $\left(x_{0}, x_{0}^{\prime}\right),\left(y_{0}, y_{0}^{\prime}\right)$ and $\left(x_{0}, y_{0}\right)$ planes. The projection onto the $\left(x_{0}, x_{0}^{\prime}\right)$ plane is, by definition,

$$
P\left(x_{0}, x_{0}^{\prime}\right)=\int \rho\left(\mathbf{Z}_{0}\right) d y_{0} d y_{0}^{\prime}=\left(\frac{1}{2 \pi \epsilon}\right)^{2} \int e^{-W_{0}} d y_{0} d y_{0}^{\prime}
$$

and in terms of the transformed coordinates,

$$
\mathbf{X}=\mathbf{X}_{0}, \quad \mathbf{Y}=\mathbf{Y}_{0}-\mathbf{C}_{0}^{\dagger} \mathbf{F}_{0}^{-1} \mathbf{X}_{0}
$$

we have

$$
W_{0}=W_{x}+W_{y}
$$

where

$$
\begin{gathered}
W_{x}=\frac{1}{2 \epsilon} \mathbf{X}^{\dagger} \mathbf{F}^{-1} \mathbf{X}=\frac{1}{2 \epsilon} \mathbf{X}_{0}^{\dagger} \mathbf{F}_{0}^{-1} \mathbf{X}_{0} \\
W_{y}=\frac{1}{2 \epsilon} \mathbf{Y}^{\dagger} \mathbf{G}^{-1} \mathbf{Y}
\end{gathered}
$$

and $\mathbf{G}$ is given by (34). Then, since the Jacobian of the transformation from $\mathbf{Y}_{0}$ to $\mathbf{Y}$ is equal to one, the projection becomes

$$
P\left(x_{0}, x_{0}^{\prime}\right)=N_{x} e^{-W_{x}}
$$


where

$$
N_{x}=\left(\frac{1}{2 \pi \epsilon}\right)^{2} \int e^{-W_{y}} d y d y^{\prime}
$$

Now, one does not have to do the integration indicated in (65) to obtain $N_{x}$, for its value also follows from the requirement that

$$
\int P\left(x_{0}, x_{0}^{\prime}\right) d x_{0} d x_{0}^{\prime}=\int \rho\left(\mathbf{Z}_{0}\right) d x_{0} d x_{0}^{\prime} d y_{0} d y_{0}^{\prime}=1
$$

Thus, defining

$$
\mathbf{f}_{0}=\mathbf{F}_{0} / D, \quad D=\left|\mathbf{F}_{0}\right|^{1 / 2},
$$

and transforming to coordinates which diagonalize $\mathbf{f}_{0}$, we find that

$$
\int e^{-W_{x}} d x_{0} d x_{0}^{\prime}=2 \pi \epsilon D
$$

and therefore

$$
P\left(x_{0}, x_{0}^{\prime}\right)=\frac{1}{2 \pi \epsilon D} e^{-W_{x}}, \quad W_{x}=\frac{1}{2 \epsilon D} \mathbf{X}_{0}^{\dagger} \mathbf{f}_{0}^{-1} \mathbf{X}_{0} .
$$

Generalizing the argument we find that the projection of the Gaussian distribution

$$
\rho(\mathbf{Z})=\left(\frac{1}{2 \pi \epsilon}\right)^{2} e^{-W}, \quad W=\frac{1}{2 \epsilon} \mathbf{Z}^{\dagger} \mathbf{E}^{-1} \mathbf{Z}, \quad|\mathbf{E}|=1
$$

on the $Z_{m}, Z_{n}$ plane is given by

$$
P\left(Z_{m}, Z_{n}\right)=\frac{1}{2 \pi \epsilon D} e^{-W}, \quad W=\frac{1}{2 \epsilon D} \mathbf{X}^{\dagger} \mathbf{f}^{-1} \mathbf{X}
$$

where

$$
\mathbf{X}=\left(\begin{array}{c}
Z_{m} \\
Z_{n}
\end{array}\right), \quad D=\left(E_{m m} E_{n n}-E_{m n}^{2}\right)^{1 / 2},
$$

and the matrix elements of $f$ are

$$
f_{11}=E_{m m} / D, \quad f_{22}=E_{n n} / D, \quad f_{12}=f_{21}=E_{m n} / D
$$

Similarly, the projection on the $Z_{m}$ axis is given by

$$
P\left(Z_{m}\right)=\left(\frac{1}{2 \pi \epsilon D}\right)^{1 / 2} e^{-W}, \quad W=\frac{Z_{m}^{2}}{2 \epsilon D}
$$

where $D=E_{m m}$. 


\section{References}

1. See, e.g., J. Powell and B. Crasemann, Quantum Mechanics, Addison-Wesley Publishing Co., p. 477 (1961).

2. L. Mirsky, An Introduction to Linear Algebra, Dover Publications, Inc., pp. 100-106.

3. C.G. Cullen, Matricies and Linear Transformations, 2nd edition, Dover Publications, Inc., pp. 31-37. 